\title{
Porcine training models for endoscopic and robotic reconstructive breast surgery: a preliminary study
}

\author{
Joon Seok Lee ${ }^{1 \#}$, Jeeyeon Lee ${ }^{2 \#}$, Yun Hyun Kim ${ }^{1 \wedge}$, Ho Yong Park ${ }^{2} \wedge$, Jung Dug Yang ${ }^{1 \wedge}$ \\ ${ }^{1}$ Department of Plastic and Reconstructive Surgery, School of Medicine, Kyungpook National University, Daegu, Korea; ${ }^{2}$ Department of Surgery, \\ School of Medicine, Kyungpook National University, Daegu, Korea \\ Contributions: (I) Conception and design: JD Yang, HY Park; (II) Administrative support: J Lee, HY Park; (III) Provision of study materials or \\ patients: JS Lee, J Lee, YH Kim; (IV) Collection and assembly of data: JS Lee, J Lee, YH Kim; (V) Data analysis and interpretation: JS Lee, J Lee; (VI) \\ Manuscript writing: All authors; (VII) Final approval of manuscript: All authors. \\ \#These authors contributed equally to this work. \\ Correspondence to: Ho Yong Park, MD, PhD. Department of Surgery, School of Medicine, Kyungpook National University, 807 Hoguk-ro, Buk-gu, \\ Daegu, 41944, Korea. Email: phy123@knu.ac.kr; Jung Dug Yang, MD, PhD, Department of Plastic and Reconstructive Surgery, School of Medicine, \\ Kyungpook National University, 130 Dongduk-ro, Daegu, 41944, Korea. Email: lambyang@knu.ac.kr.
}

\begin{abstract}
Background: With recent advances in surgical techniques, minimally invasive methods for reconstructive breast surgery are being investigated. To enhance surgical proficiency through training and minimize predictable complications before human application, we conducted a preliminary experimental study of reconstructive breast surgery using a porcine model.

Methods: Between February and March 2019, four 3-month-old pigs underwent a bilateral mastectomy and immediate breast reconstruction with a latissimus dorsi (LD) flap or silicone implants. After performing the mastectomy by dissecting the pectoralis profundus in the subcutaneous plane, the pig was placed in the decubitus position, and ultrasound-guided marking was used to design the LD flap. The thoracodorsal artery was marked, and a 4-cm incision was made on the midaxillary line. An additional endoscopic incision was made in the inferior margin of the LD flap; a 2-hole approach was used for endoscopic LD flap elevation. In the silicone implant model, a silicone implant (Allergan, smooth, round type, $90 \mathrm{cc}$ ) was placed using a single incision $(4-5 \mathrm{~cm})$.

Results: Eight mastectomies followed by breast reconstruction with LD flap elevation or silicone implant models were performed on four pigs. Serious complications, such as active bleeding, did not occur. However, heat dispersion to the skin flap that became thinner by endoscopic dissection caused a second-degree burn in one pig.

Conclusions: This preliminary study of endoscopic or robot-assisted minimally invasive reconstructive breast surgery demonstrates that a porcine training model is a highly valuable experimental model for surface anatomy verification, incision plan selection, instrument selection, operator proficiency enhancement, and complication prevention.
\end{abstract}

Keywords! Reconstructive breast surgery; porcine training model; endoscopic and robotic surgery

Submitted Jun 16, 2021. Accepted for publication Jul 23, 2021.

doi: $10.21037 / g s-21-398$

View this article at: https://dx.doi.org/10.21037/gs-21-398

\footnotetext{
^ ORCID: Joon Seok Lee, 0000-0002-1580-0487; Jeeyeon Lee, 0000-0003-1826-1690; Yun Hyun Kim, 0000-0002-9137-0909; Ho Yong Park, 0000-0002-4380-0089; Jung Dug Yang, 0000-0002-9121-3604.
} 


\section{Introduction}

Minimally invasive surgery is employed in various fields to minimize surgical incisions. Various instruments are used, including laparoscopic or endoscopic devices, and recently the trend has moved toward robotic surgery (1). Such trends have also influenced breast surgery, even extending to research on breast reconstruction. The goal of breast reconstruction, in particular, is not only to restore breast volume after skin-sparing mastectomy but is also to create the aesthetic, feminine shape of the breast. However, breast surgery is somewhat different from surgery in other solid organs due to its anatomical structure. Most laparoscopic and robotic surgeries conducted to date have been related to solid intraperitoneal organs. Breast reconstruction after mastectomy for breast cancer is different because the breast is a unique anatomical structure that is highly visible and has a round, smooth outline; therefore, determining the appropriate position of the incision and approach is important. Since the location and shape of the reconstruction site after skin-sparing mastectomy is variable rather than fixed, it is difficult to reconstruct a similar shape when replacing the breast tissue.

Therefore, to use endoscopic or robotic surgery for breast reconstruction, a preliminary experimental model for surgical training that enables surgical planning to minimize complications is very important (1-6).

Thus, the present study proposes a preliminary porcine model, which has anatomical similarities to humans, for breast reconstruction after skin-sparing mastectomy that can be used in the planning process for endoscopic or robotassisted surgery to determine the incision location and minimize the length of the incision. We present the following article in accordance with the ARRIVE reporting checklist (available at https://dx.doi.org/10.21037/gs-21-398).

\section{Methods}

\section{Animals}

Four 3-month-old female pigs (Duroc Jersey, large white; $35-40 \mathrm{~kg}$ ) were used in the present experimental non-survival study. Experiments were performed under a project license (ISI Protocol No. 004, MIC-IACUC-2019-01) granted by national ethics board of the Institutional Animal Care and Use Committee (IACUC), in compliance with Korean IACUC institutional guidelines for the care and use of animals.

The four pigs underwent eight model surgeries on both sides from February to April 2019. After confirming the surface anatomy, they underwent a mastectomy followed by breast reconstruction using the latissimus dorsi (LD) flap model and using the silicone implant model. Preoperatively, pigs fasted for 16 hours and had free access to water. Anesthesia was induced by premedicating with an intramuscular injection of Zoletil ( $4 \mathrm{mg} / \mathrm{kg}$, Virbac). Five minutes later, an intramuscular injection of atropine $(0.05 \mathrm{mg} / \mathrm{kg}$, Jeil) and flunixin meglumine $(1 \mathrm{mg} / \mathrm{kg}$, EagleVet) was administered. Anesthesia was maintained with isoflurane $2-2.5 \%$. At the end of the procedure, the pig was sacrificed with potassium chloride intravenous injection after induction of deep anesthesia by high-dose isoflurane (5\%, $7 \mathrm{~min})$.

\section{Instruments}

We used the standard endoscopic setup with a light source, $10 \mathrm{~mm}$ and $5 \mathrm{~mm}$ 30-degree endoscopes, a video camera, a video monitor, and a video recorder. The usual instrument set for flap surgery and standard incision instruments (forceps, cautery, scissors, and a LigaSure Small Jaw device) were also used (5). We used the LigaSure Small Jaw [Medtronic (formerly Covidien), Minneapolis, MN, USA] device for dissection and cutting. We first practiced using a surgical glove-port and then used the single port (The Oneport, KDL Health Care, Korea) for the procedure.

\section{Operating room settings}

The pig was first placed in a supine position with the operator standing on one side and an assistant on the other to conduct a bilateral mastectomy model. Next, a LD flap model was conducted in the bilateral decubitus position. We then placed the pig in the supine position and performed breast reconstruction using the LD flap and a silicone implant. In each position, a video monitor was placed cranial or caudal to the pig so that both the operator and the assistant could see.

\section{Mastectomy training in the porcine model}

To define the mastectomy model in the pig, we marked the regions corresponding to the human pectoralis major muscle, which were the pig pectoralis superficialis (red) and pectoralis profundus (blue) (Figure $1 A$ ). We designed the model by verifying the pectoralis margin using ultrasound-guided marking (Figure 1). Before marking, we first confirmed via ultrasound that the muscle became thinner as it reached the boundary. We set the incision location as the midaxillary line to allow for mastectomy, axillary lymph node sampling, and 

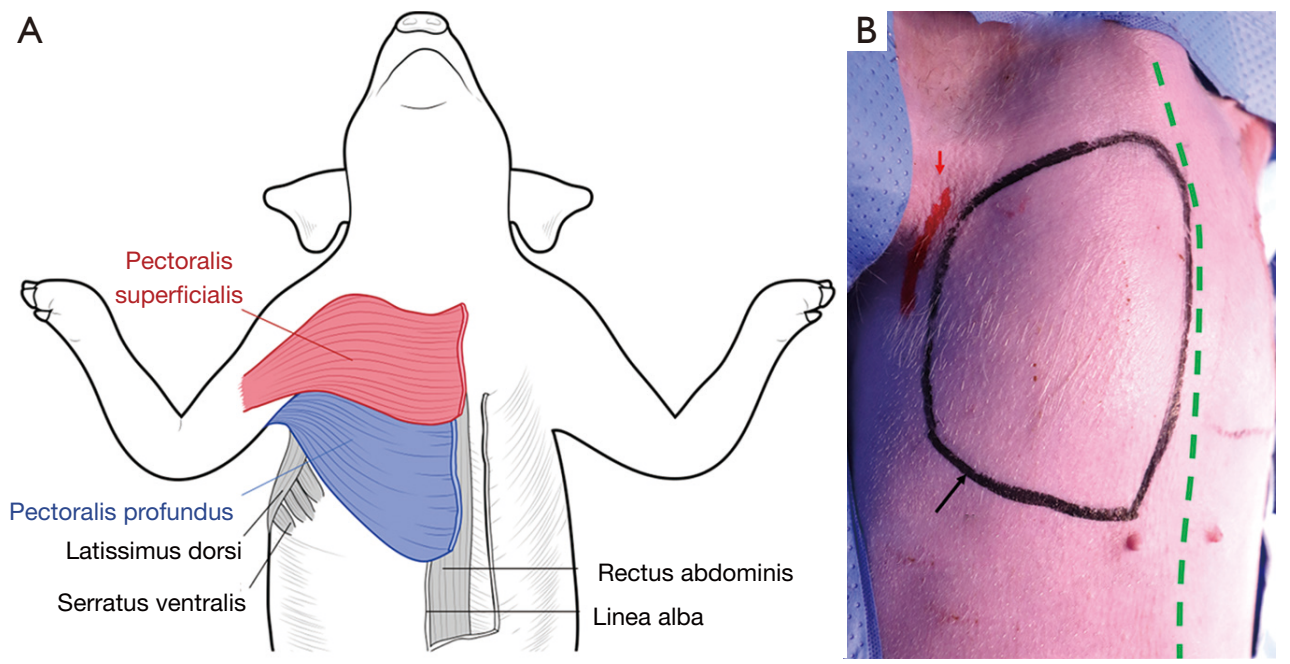

Figure 1 Ventral surface anatomy for the mastectomy model. (A) Ventral muscles of the porcine model—pectoralis superficialis (red), pectoralis profundus (blue). (B) Ultrasound-guided surface anatomy of the pectoralis muscle.
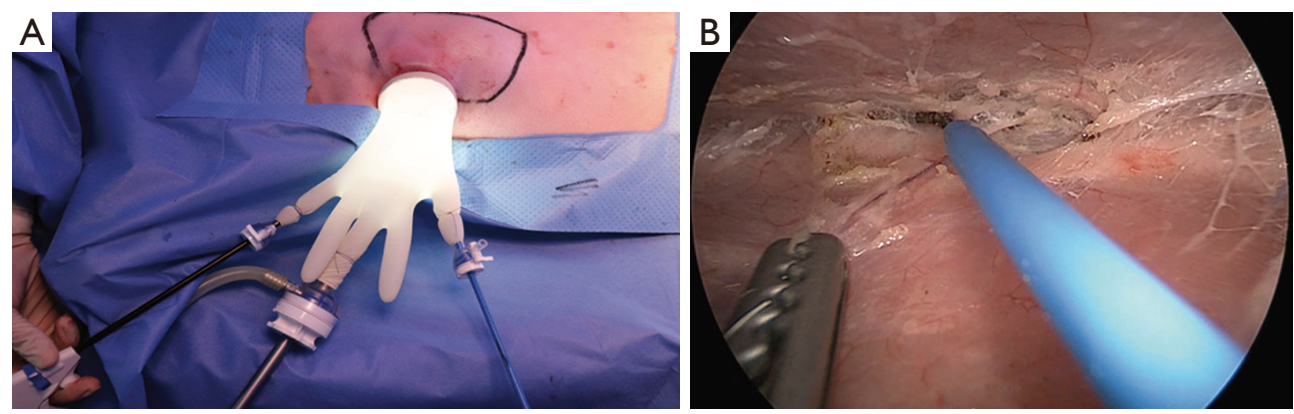

Figure 2 Intraoperative findings. (A) Endoscopic device settings in the porcine model. (B) Intraoperative findings during endoscopic dissection for the latissimus dorsi flap elevation.

access to the LD flap for immediate reconstruction (Figure 1B).

After the design was complete, we made a $4-5 \mathrm{~cm}$ incision along the axillary line and undermining within about $2-3 \mathrm{~cm}$. We used a wound retractor to enable an approach to the area and a surgical glove-port to use as a multiport, with a camera port in the middle and monopolar and forceps on each side, and then conducted inflation using $\mathrm{CO}_{2}$ gas (9-10 $\mathrm{mmHg}$ ) (Figure $2 A$ ). We used endoscopy to reach below the skin flap and dissect carefully (Figure $2 B$ ). Well-organized cooperation between the camera port, controller, and the operator is necessary. We then simultaneously performed dissection and bleeding control up to the marking using monopolar electrosurgery. A single incision mastectomy model was possible in the porcine model. The mastectomy was performed by dissecting the pectoralis profundus in the subcutaneous plane.

\section{LD flap harvest in the porcine training model (Video 1)}

We first verified the surface anatomy using the pig LD muscle as the landmark. After decubitus positioning of the pig, we designed a parallelogram using the midpoint of the line connecting the scapular tip and olecranon and the middle $1 / 3$ point of the porcine spine, which can be assumed to be the region where the LD muscle is located. We drew the incision line for the LD approach about 4-5 cm along the midaxillary line (Figure 3).

The dominant pedicle is composed of (from the axillary artery via subscapular artery) the thoracodorsal artery and a pair of Vena comitans (7). We confirmed and marked the thoracodorsal vessels using Doppler ultrasound and made a $4-5 \mathrm{~cm}$ incision along the midaxillary line passing through the subcutaneous fat layer and submuscular layer 

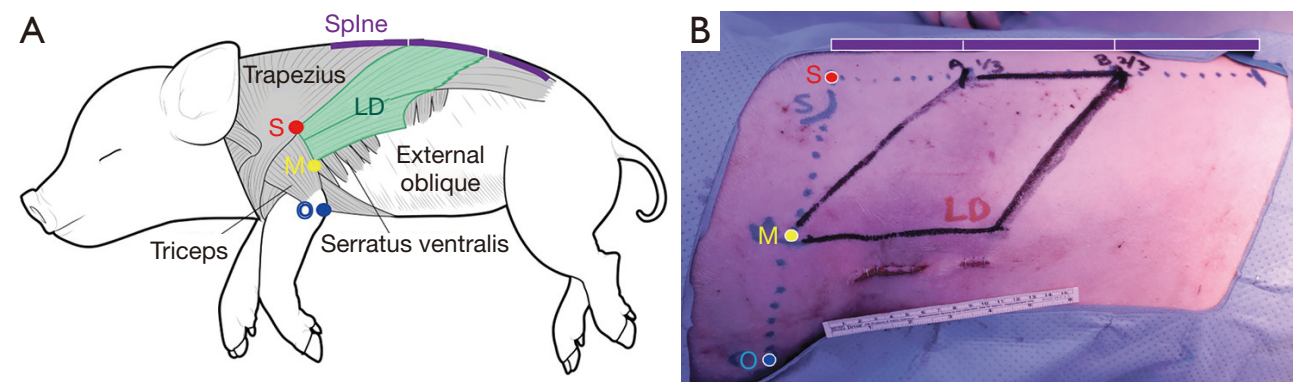

Figure 3 Lateral surface anatomy for latissimus dorsi flap model. (A) Lateral muscles of the porcine model. (B) Surface anatomy: LD in the porcine model. LD, latissimus dorsi; S, scapular tip; O, olecranon; M, midpoint.
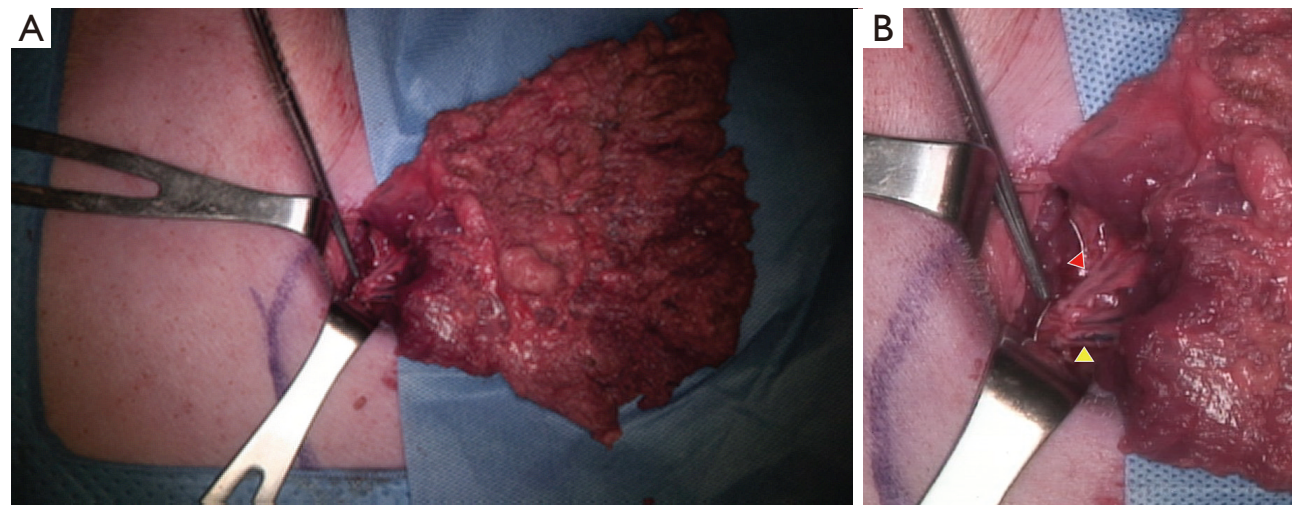

Figure 4 Latissimus dorsi flap in the porcine model and thoracodorsal vessels. (A) Elevated latissimus dorsi flap. (B) Isolated pedicles of the latissimus dorsi flap (red arrowhead: transverse pedicle; yellow arrowhead: longitudinal pedicle).

and through the fat and panniculus down to the muscle. Next, to make it easier to approach the inferior margin of the LD flap, we performed an additional small incision in the inferior margin and tried a double port approach for endoscopic LD flap elevation (Figure 3). Although it was difficult to cut the inferior margin of the LD muscle using the midaxillary incision alone, it was possible to utilize the auto-clamping device (Thunderbeat, Olympus Medical Systems Corp, Tokyo, Japan) for double incision. Then, we dissected and isolated the LD from the trapezius and serratus ventralis in the upward direction. We confirmed the dissected pedicle, the thoracodorsal artery, and the vein and carefully moved it to the location of the breast without injury for immediate breast reconstruction using the LD flap in the porcine model (Figures 4,5).

\section{Implant-based reconstruction training in the porcine model}

We inserted a 90-cc round type silicone implant (Natrelle 410 , Allergan) using the same incision created in the mastectomy model. The results of right breast reconstruction are shown in Figure 6.

\section{Results}

Eight mastectomies followed by breast reconstruction using LD flap elevation or silicone implant insertions were performed on four porcine models. In the implant model, we designed an incision at least 4-5 cm in length for implant insertion. Serious complications, such as active bleeding, were not found. However, heat dispersion to the skin flap that became thinner from dissection by the endoscopic approach caused a second-degree burn in one pig (Figure 7).

\section{Discussion}

In Asians, small to moderate breast volume is the most common, so breast reconstruction is most possible with $\mathrm{LD}$ flap as an autologous tissue. If the volume is insufficient, a 

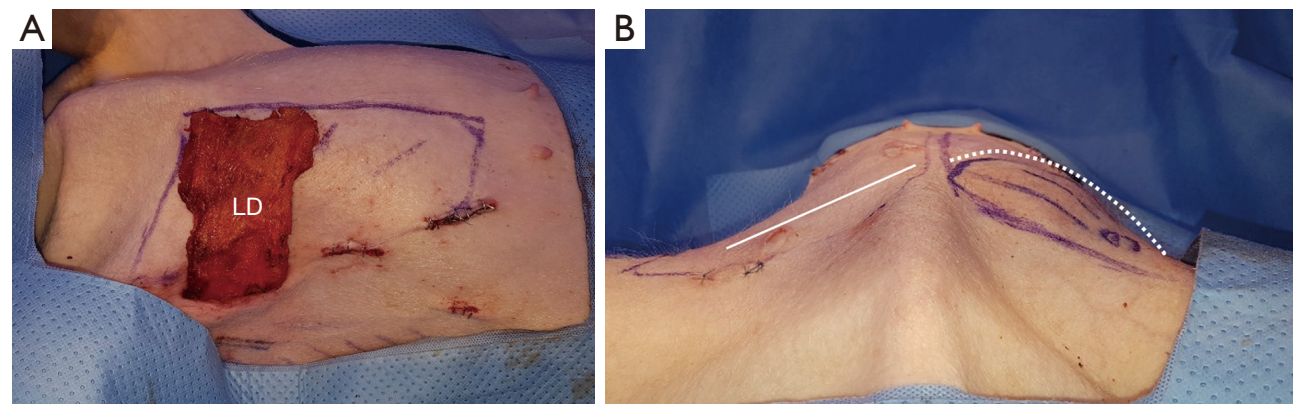

Figure 5 Immediate breast reconstruction using the LD flap in the porcine model. (A) Transpositioned LD flap (LD flap mean weight: $77 \mathrm{~g}$ ). (B) Immediate postoperative findings of right breast reconstruction (white dotted line: breast reconstruction using LD flap). LD, latissimus dorsi.
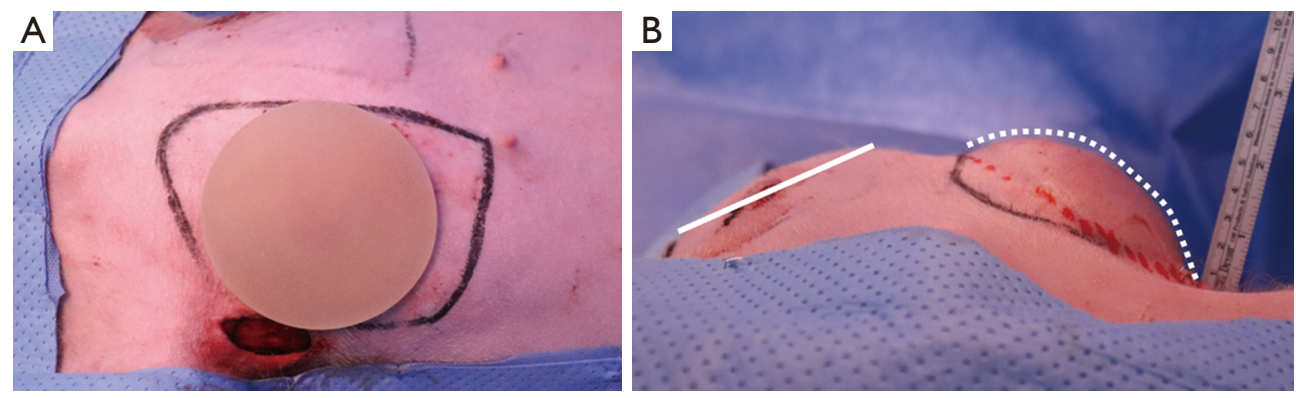

Figure 6 Immediate implant-based breast reconstruction in a porcine model. (A) Silicone implant (Allergan, smooth, round type, 90 cc), (B) immediate postoperative findings of right breast reconstruction (white dotted line: breast reconstruction using a silicone implant).

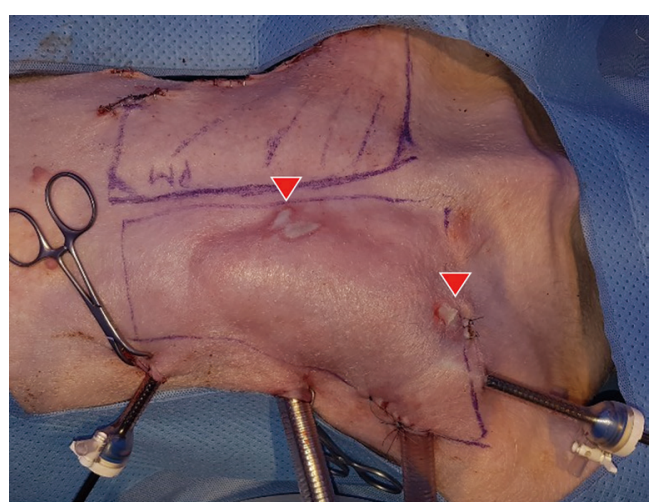

Figure 7 Complications of the first case in the porcine mastectomy model. Necrosis on the left breast area (red arrowhead).

small implant is additionally inserted to satisfy the natural shape and symmetry of the reconstructed breast, and it is reported that the functional outcome is also reliable. This study established a porcine training model to be used before clinical application of skin-sparing mastectomy and immediate breast reconstruction surgery in Asians for each of the steps of surface anatomy, mastectomy, and LD flap and silicone implant breast reconstruction. Such a preliminary large animal training model is highly crucial for exploring the various aspects of surgical planning, selection of devices according to the surgical method, number and arrangement of ports, and possible complications before performing endoscopic or robot-assisted breast surgery using a small incision. Therefore, we believe that the porcine model used in this study has high value as a model for surgical training before the clinical application of endoscopic or robot-assisted breast surgery.

There is an increasing need for immediate breast reconstruction after breast mastectomy in breast cancer. Since the aesthetic outcome of breast reconstruction is an important factor along with oncological safety, postsurgery aesthetics, including shape and scarring, should be sufficiently taken into consideration. Recently, many studies have focused on reducing scarring and enhancing surgical precision, and various methods and devices for minimal surgical incision have been proposed (8). Moreover, it has been reported that patients' postoperative pain can be reduced by performing endoscopic surgeries. In breast 
reconstruction using the $\mathrm{LD}$ flap, the incision length is within $5 \mathrm{~cm}$ for endoscopic or robotic surgery; however, it is $15 \mathrm{~cm}$ or greater for conventional surgery. The advantage of endoscopic and robotic reconstructive breast surgery is quite clear, and the benefits are believed to be comparable to those of endoscopic or robotic abdominal surgery $(9,10)$. If the incidence of other complications in endoscopic or roboticassisted mastectomy and immediate breast reconstruction with LD flap or silicone implant is not higher than that in conventional surgery, then it can be an excellent alternative that boosts patient satisfaction by minimizing scarring and postoperative pain. For the incidence of complications to be lower in endoscopic or robotic surgery than in conventional methods, the proficiency of the surgeons is highly critical. Since high proficiency requires sufficient practice before clinical application, we believe that designing a large animal experimental model for training is necessary (1-3).

Regarding reconstructive surgery, an endoscopymediated harvest of the LD muscle flap was first reported by Friedlander and Sundin in 1995 (9). Fine et al. and Cho et al. reported that when performing a lower limb defect reconstructive surgery, using endoscopy for harvesting the LD muscle is both feasible and productive $(11,12)$. In addition, Avram et al. and Bodin et al. confirmed the anatomy using a large animal model and designed a breast reconstruction model prior to clinical application of the free flap $(3,4)$. Since then, there have been insufficient reports about applying this method to oncologic breast reconstruction surgery, and the method failed to gain popularity in breast reconstruction. The breast is an anatomical structure, unlike solid organs, that is exposed on the outside, has a rounded shape, and its skin flap is not fixed after mastectomy (12). Therefore, it is difficult to maintain the long length of the operation while considering the convex shape of the chest wall. Consistent with the current trend toward less aggressive methods of reconstructive breast surgery, mastectomy and LD muscle harvesting via minimally invasive surgery have recently emerged as new techniques of reconstructive breast surgery (13).

While pigs and dogs are the most commonly used large animal models, using pigs is recommended since dogs have a LD flap vasculature distinct from the human vasculature and cannot be used (14). The human pectoralis major muscle is located below the breast parenchyma and shares anatomical similarities with the porcine pectoralis superficialis and profundus. Except for the fact that the human LD muscle originates from the lower six thoracic vertebrae, lumbar fascia, and ilium, whereas the porcine muscle originates from the last four ribs excluding one, the anatomy of the porcine LD muscle is very similar to the human anatomy. The primary arterial supply comes from the thoracodorsal artery, a branch of the subscapular artery, which eventually becomes the subclavian artery (15). Such similarities in gross anatomy between porcine models and humans, along with a body size suitable for devices used on humans, make it a valuable animal experimental model. Furthermore, pigs are commonly used in various large animal experiments because their panniculus carnosus does not contribute to the blood supply in the skin, making it more suitable than other animal models for preliminary experiments (15).

In mastectomy, the breast tissue must be excised using a single incision, but since the chest wall is convex-shaped, and the breast also has an anterior protruding tear-drop shape, selecting the appropriate incision location and devices is highly critical. Moreover, in LD breast reconstruction, access to the medial part of the breast is often difficult, and a long scar is frequently created in the donor site. The endoscopic or robotic approach enables surgery in a large operative field using only a small incision and is expected to shorten the length of the hospital stay by reducing early drain removal and postoperative pain (13). Most importantly, this method enhances the aesthetic outcome and reduces donor site morbidity by minimizing visible scarring after surgery. Several reports have reported that the endoscopic method is not significantly different from conventional methods regarding seroma occurrence (13).

Taken together, we can conclude that the endoscopic or robot-assisted surgical method has clinical value if the complication rate is low and the donor site morbidity and postoperative pain are effectively reduced compared to conventional methods. Additionally, since the endoscopic or robot-assisted surgical method allows immediate reconstruction of small-to-moderate Asian breasts where volume replacement is possible with a muscle flap alone, defining the appropriate indications to achieve the desired effect is critical (9).

However, even if the appropriate indications are defined, since the endoscopic or robot-assisted surgical method requires more time from preoperative setup to flap harvest than conventional methods, sufficient preoperative practice and planning, proper selection of surgical tools, and surgical proficiency are indispensable. Furthermore, in our experimental study, heat dispersion to the skin flap that becomes thinner from dissection by the endoscopic approach caused a second-degree burn in one pig. For this 
reason, extensive training similar to actual clinical practice is required before clinically applying the endoscopic or robotassisted approach. In the first mastectomy performed in this study, necrosis of the skin flap also occurred (Figure 7). Thus, the key to minimizing the possible occurrence of such complications is to use a porcine training model prior to human application for preoperative design, selecting the tools suited for the operator, and planning and mastering the surgical technique to reduce the operation time and possible complications.

Although traditional harvesting has a straightforward learning curve, it has been reported that the endoscopic or robot-assisted harvest must have an additional learning curve to yield a similar outcome with less morbidity. ${ }^{5}$ Therefore, an established training model is required to learn and master the endoscopic or robot-assisted surgery, and using a porcine model to check the surface anatomy and conduct skin-sparing mastectomy, LD harvesting, and silicone implant is ideal. We believe that the porcine training model developed in the present study can be a very useful training model for clinical application in Asian patients.

\section{Conclusions}

We conducted a preliminary experimental study of endoscopic or robot-assisted reconstructive breast surgery as a minimally invasive surgical method and report that a porcine training model, which is anatomically similar to the human model, is a highly valuable and reliable experimental animal model for surface anatomy verification, incision plan selection, instrument selection, operator proficiency enhancement, and complication prevention.

\section{Acknowledgments}

Funding: None.

\section{Footnote}

Reporting Checklist: The authors have completed the ARRIVE reporting checklist. Available at https://dx.doi. org/10.21037/gs-21-398

Data Sharing Statement: Available at https://dx.doi. org/10.21037/gs-21-398

Conflicts of Interest: All authors have completed the ICMJE uniform disclosure form (available at https://dx.doi. org/10.21037/gs-21-398). The authors have no conflicts of interest to declare.

Ethical Statement: The authors are accountable for all aspects of the work in ensuring that questions related to the accuracy or integrity of any part of the work are appropriately investigated and resolved. Experiments were performed under a project license (ISI Protocol No. 004, MIC-IACUC-2019-01) granted by national ethics board of the Institutional Animal Care and Use Committee (IACUC), in compliance with Korean IACUC institutional guidelines for the care and use of animals.

Open Access Statement: This is an Open Access article distributed in accordance with the Creative Commons Attribution-NonCommercial-NoDerivs 4.0 International License (CC BY-NC-ND 4.0), which permits the noncommercial replication and distribution of the article with the strict proviso that no changes or edits are made and the original work is properly cited (including links to both the formal publication through the relevant DOI and the license). See: https://creativecommons.org/licenses/by-nc-nd/4.0/.

\section{References}

1. Foo CC, Yeung CK, Sreedhar B, et al. Robotic-Assisted Transrectal Cholecystectomy in a Porcine Model. Surg Innov 2019;26:436-41.

2. Hoffman MS, Spiess PE. Simulated management of urinary tract injury during robotic pelvic surgery utilizing the porcine model. J Robot Surg 2019;13:289-92.

3. Avram TE, Muntean M, Janko B, et al. Free-flap breast reconstruction on experimental porcine model. Injury 2019;50 Suppl 5:S123-5.

4. Bodin F, Diana M, Koutsomanis A, et al. Porcine model for free-flap breast reconstruction training. J Plast Reconstr Aesthet Surg 2015;68:1402-9.

5. Friedlander LD, Sundin J, Bakshandeh N. Endoscopy mastectomy and breast reconstruction: endoscopic breast surgery. Aesthetic Plast Surg 1995;19:27-9.

6. Wang S, Liu Y, Fang D, et al. The miniature pig: a useful large animal model for dental and orofacial research. Oral Dis 2007;13:530-7.

7. Ghetu N, Mojallal AA, Huber D, et al. Endoscopicassisted harvest of pedicled and free latissimus dorsi muscle in pigs. Timisoara Med J 2010;60:145-53.

8. Lee MA, Miteff KG. The scarless latissimus dorsi flap provides effective lower pole prosthetic coverage in 
breast reconstruction. Plast Reconstr Surg Glob Open 2014;2:e147.

9. Missana MC, Pomel C. Endoscopic latissimus dorsi flap harvesting. Am J Surg 2007;194:164-9.

10. Chen YQ, Tao JW, Su LY, et al. Cooperative robot assistant for vitreoretinal microsurgery: development of the RVRMS and feasibility studies in an animal model. Graefes Arch Clin Exp Ophthalmol 2017;25 5:1167-71.

11. Cho BC, Lee JH, Ramasastry SS, et al. Free latissimus dorsi muscle transfer using an endoscopic technique. Ann Plast Surg 1997;38:586-93.

12. Fine NA, Orgill DP, Pribaz JJ. Early clinical experience

Cite this article as: Lee JS, Lee J, Kim YH, Park HY, Yang JD. Porcine training models for endoscopic and robotic reconstructive breast surgery: a preliminary study. Gland Surg 2021;10(8):23462353. doi: 10.21037 /gs-21-398 in endoscopic-assisted muscle flap harvest. Ann Plast Surg 1994;33:465-9; discussion 469-72.

13. Silva Vergara C, Yoon T, Font J. A minimally invasive strategy for breast reconstruction: Latissimus dorsi muscle flap harvest by single-port endoscopy. J Breast Cancer Res Adv 2017. doi: 10.16966/jbcra.103.

14. Yang D, Morris SF. Differences in intramuscular vascular connections of human and dog latissimus dorsi muscles. Ann Thorac Surg 1999;67:489-93.

15. Millican PG, Poole MD. A pig model for investigation of muscle and myocutaneous flaps. Br J Plast Surg 1985;38:364-8. 